\title{
Research of Long-Term Development of Integrated Energy Systems with Combinatorial Modelling Methods
}

\author{
Valery A. Stennikov ${ }^{1}$, Valeriy I. Zorkaltsev ${ }^{2}$, Evgeny A. Barakhtenko ${ }^{3}$, Aleksey V. Edelev ${ }^{4}$ \\ Melentiev Energy Systems Institute of Siberian Branch of the Russian Academy of Sciences, \\ Lermontov str., 130 \\ Irkutsk, Russia \\ ${ }^{1}$ E-mail: sva@isem.irk.ru \\ ${ }^{2}$ E-mail: zork@isem.irk.ru \\ ${ }^{3}$ E-mail: barakhtenko@isem.irk.ru \\ ${ }^{4}$ E-mail: flower@isem.irk.ru
}

\begin{abstract}
The paper describes a combinatorial modelling approach to the research of integrated energy systems development. The idea of the approach is to model a system development in the form of a directed graph with its nodes corresponding to the possible states of a system and arcs characterizing the possibility of transitions from one state to another. It allows us to simulate a long-term process of system development under various possible conditions, and determining an optimal system development strategy.
\end{abstract}

Keywords: Combinatorial modelling, integrated energy system, long-term development, decision support.

\section{Introduction}

In the modern conditions, the transition to integrated energy systems becomes more actual because of the appearance of new technologies and equipment, new requirements for energy supply systems and changing their functioning conditions. The integrated energy systems represent a combination of electric, heat, cooling and gas supply systems. The studies have shown that the joint consideration of energy supply systems of different types during researching their development and operation problems provides a number of advantages in comparison with the traditionally isolated consideration of these systems [1-3].

The problem of developing integrated energy systems is to determine the optimal combination of generation, transformation and storage technologies as well as a network structure. This combination must ensure the satisfaction of the forecasted energy demand. The deterministic or probabilistic methods are distinguished to solve the problem [4]. In deterministic methods, the variables affected the amount of energy supply system investments are considered to be known $[5,6]$. Stochastic methods take into account the various energy system uncertainties associated with energy markets functioning and the energy generation volatility increment $[7,8]$.
Consideration of multiple scenarios enables the analysis of solutions regarding the impact of input data uncertainties [9]. Scenarios are key tools in energy development research and decision making in the face of an uncertain future [10]. Some scenario techniques improve the building and use of scenarios for dealing with the dual challenge of complexity and uncertainty [11]. The very broadly used approach is to use the judgment of the group of decision makers and/or experts to create a small number of scenarios that can effectively cover the space of feasible development alternatives. However, this approach is subject to human cognition biases and social effects at play in the group, which may interfere to explore the uncertainty [12] Alternative approaches ensure diversity of scenarios constructed [13] or focus on determining the vulnerability of strategies to the uncertainty [14], or by combining both [15].

In [16] the payoff matrix technique is presented for solving optimization problems under uncertainty. Special Wald, Laplace and Subjective probability estimations had been applied in that technique. Stochastic variables are used to represent the probabilistic characteristics of uncertain input data that are not precisely known (or are not known at all). This input data inaccuracy necessitates the consideration of several possible combinations of scenarios and external 
conditions to form a payoff matrix. The choice of a rational alternative is made after the payoff matrix analysis.

In many scenarios that depend upon mathematical models the main modelling efforts are directed to produce cost optimal results and too little attention is given to the implications of plausible alternatives [11]. The Modelling to Generate Alternatives approach [17] focuses on the diversity of outputs and changes the underlying structure of the mathematical model to search the near-optimal region of the output space for alternative outputs that are very different in the decision space. The combinatorial modelling [18] generates various scenarios based on different model input assumptions and emphasizes on the diversity of pathways that lead to a given type of output.

The combinatorial modelling is a framework in the language from [19] where distinguish is made between the three terms: model, model generator, and framework. Models are concrete representations of realworld systems to answer clearly defined research questions. Models can be built from model generators that allow building models with a certain analytical and mathematical approach. Finally, a framework can be understood as a structured toolbox including subframeworks and model generators as well as specific models.

In this paper, the applicability of the combinatorial modelling framework is considered for studying the long-term development of intelligent integrated energy systems. This framework has been tested in the study of energy security provision in Russia [20] and Vietnam [21].

\section{The combinatorial modelling framework}

The basic concept of combinatorial modelling is to represent the development of an object belonging to the considered system in the form of a directed graph. Nodes of the graph are associated with object states at a particular time interval $t=1, \ldots, T$, where $T$ is the number of time intervals. The arcs characterize possible transitions from one state to another. All development paths of each object start from the single initial state.

Objects as structural units of the considered system may be enterprises of the energy sector or their groups, consumers of energy resources or in general their categories. The degree of aggregation of objects depends on the task to be solved and the available information. Within the combinatorial modelling framework, it is possible to unite objects in a natural way. A collection of several objects in one task can represent a single object while considering the problems of higher level.

The graph containing possible states and transitions of whole system development is constructed by combining various states of objects at the same time intervals and the possible transitions between states at the same neighbour time intervals. The term "combinatorial modelling" is used because of the combinatorial nature of rules that set linkage between the object development graphs and the system development graph.

The first part of the combinatorial modelling framework consists of algorithms to select the valid options of the system development from possible ones. A subgraph of admissible options of the system development is separated from the constructed graph of the possible development of the system. This subgraph consists of such possible states and transitions of system development that meet the constraints described below.

The second part consists of algorithms used to find out the optimal and the entire set of suboptimal trajectories of system development in the given set of admissible trajectories of development of this system. Close-to-optimal or suboptimal trajectories are of great interest because of the inaccuracies of cost criterion computation [22]. Also the analysis of differences of near-optimal solutions provides valuable information for the decision maker when adapting the optimal solution to reality [23]. The optimal and suboptimal system development trajectories are determined by the dynamic programming approach [24], for example, as a result of minimizing the system development and functioning costs criterion.

The combinatorial modelling stages described above can help to determine the proper development directions for a system considering the system development problem in the one-criterion formulation and in the deterministic conditions. The ability to obtain the full set of suboptimal trajectories is important as a way to overcome uncertainty in the initial data.

The combinatorial modelling framework allows:

- To take into account explicitly the discreteness of options for the system objects development,

- To take into account explicitly the multi-stage character of decision-making process,

- To use other models to assess a system state validity,

- To determine the entire set of suboptimal system development paths in deterministic conditions and under uncertainty conditions. 


\section{The evaluation of a possible system state feasibility in the combinatorial modelling framework}

If the number of the possible state of the system at time interval $t$ is denoted as $d_{t}$ and a possible transition between system states with numbers $d_{t-1}$ and $d_{t}$ as $\left(d_{t-1}, d_{t}\right)$ then not all possible system states $d_{t}$ and transitions $\left(d_{t-1}, d_{t}\right)$ are feasible. Some transitions are removed due to the constraints that come from the objects nature. Possible system states and transitions that satisfy system-wide constraints (logical and balance conditions) as well as constraints on development dynamic are called feasible.

\subsection{System-wide constraints}

There are two types of system-wide constraints.

\subsubsection{Logical conditions}

Some alternatives of an object development can be realized only with particular alternatives the other objects development. Logical conditions can be specified as lists of incompatible states and transitions or, on the contrary, as the whole set of compatible ones.

\subsubsection{Balance and other constraints}

These are constraints on the limited use of resources for each time interval and for each transition between them. Such constraints can be defined as balance equations or inequalities:

$$
\begin{gathered}
g_{t}\left(d_{t}, x\right) \leq 0, \\
f_{t}\left(d_{t-1}, d_{t}, y\right) \leq 0, t=2, \ldots, T,
\end{gathered}
$$

where $g_{t}, f_{t}$ are some vector functions that depend on the numbers of states $d_{t}$ and transitions $\left(d_{t-1}, d_{t}\right)$ as well as on special additional variables vectors $x$ and $y$. If the set of Eq. (1) has a solution with regard to the vector $x$ then that state with the number $d_{t}$ is feasible on balance constraints. The Eq. (1) for fixed $d_{t}$ form the set of inequalities with respect to the vector $x$ on the basis of information characterizing the particular objects for given system state with number $d_{t}$. The vector of variables $x$ can include, for example, the intensity of technological facilities use for the system state with the number $d_{t}$ at the time $t$.

The set of in Eq. (2) is constructed similarly. If the system of Eq. (2) is consistent, then the transition $\left(d_{t-1}, d_{t}\right)$ is feasible on balance constraints.

\subsection{Development dynamic constraints}

The system states and transitions that remain after the screening according to system-wide constraints must be checked from the point of view of their compatibility in time. A state with adjacent transitions is removed if where is no path from given state with the number $d_{t}$ to any system state with a number $d_{T}$. Also an unreachable state with adjacent transitions is removed if where is no path from the initial state with the number $d_{1}$ to the given state with the number $d_{1}$.

\section{Construction and analysis of an integrated energy system development options with combinatorial modelling}

\subsection{Principles of decomposition of an integrated energy system into components}

An integrated energy system is a complex system that consists of a set of subsystems and a set of their elements. An integrated energy system includes energy supply systems of different types (electric, heating, gas and other systems) which can be considered as subsystems of the integrated energy system. The information and communication system is also a subsystem of the integrated energy system. Each subsystem contains its own set of elements. These elements can be grouped according to the following energy functions: production, transport, distribution and consumption. In turn, each element has its own set of equipment according with the energy functions and the type of the energy supply system.

In order to carry out combinatorial modelling, it is necessary to determine the objects of the integrated energy system and to form their states. A set of these objects has to describe correctly the ways of development of the integrated energy system to choose among them the most optimal way. Objects can be one of the following:

- Subsystems;

- Groups of elements (according to the performed energy functions);

- $\quad$ Elements.

\subsection{The evaluation of feasibility of an integrated energy system state in the combinatorial modelling framework}

The model of the integrated energy system functioning [2] is of the second type of system-wide constraints. The feasibility of the possible state of the integrated energy system is determined by the permissibility of the 
operation mode of the heat and power supply systems under the given conditions.

\subsection{The approximate scheme for the construction and analysis of development options of integrated energy systems}

The first step of the combinatorial modelling approach is to describe the basic scenario of energy development to investigate as a graph with one node for each time moment (Fig. 1). These nodes contain essential information to create new possible states of the considered integrated energy system.

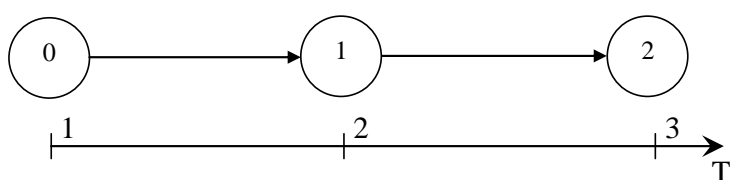

Fig. 1. Basic scenario of the integrated energy system development.

In the second step, the infrastructure of the integrated energy system is divided into several components by territorial or industrial criteria. For each component, a development graph is built. It contains changes in the energy facility parameters at the time period considered. The development graphs of two energy facilities are shown in Fig. 2. The source nodes corresponding to moment 0 do not have numbers because they will not participate in the next construction of the integrated energy system.

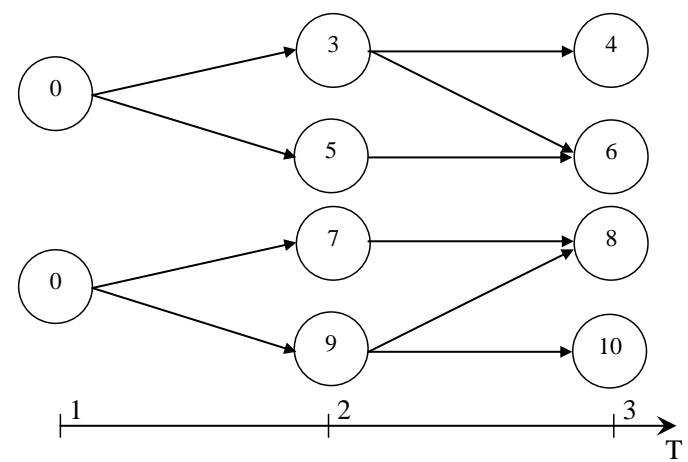

Fig. 2. Development graphs of 2 energy facilities.

The third step is combining data of the reference graph with information on the different components of graphs belonging to the same moment in time. This results in the set of possible states of the integrated energy system for each moment in time.

The states (nodes) of the modelled system are linked by transitions (arcs) to form an energy system development graph.
The integrated energy system development graph shown in Fig. 3 is constructed by means of combination of nodes and arcs of the graphs in Fig. 1 and Fig. 2. The number of generated possible integrated energy system state is shown inside the circle in Fig. 3. The numbers above the circle are combinations of the graph nodes in Fig. 1 and Fig. 2. The beginning of all paths in the generated integrated energy system development graph is a common initial node at moment 1 .

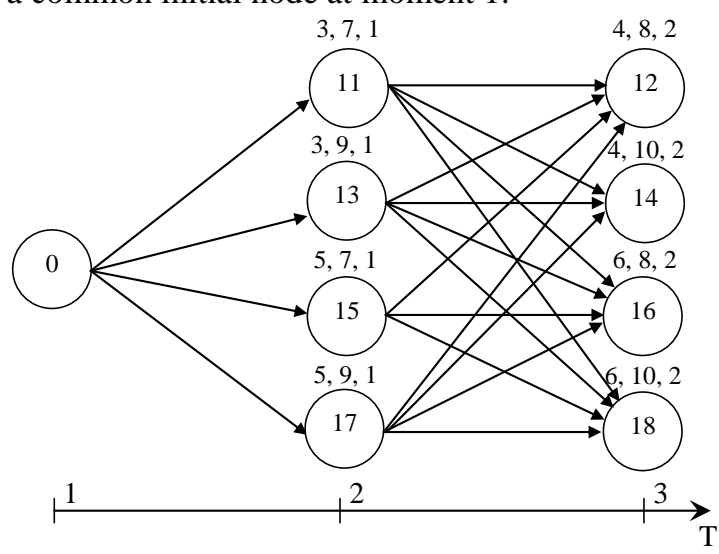

Fig. 3. Integrated energy system development graph.

The fourth step is to check the feasibility of nodes and arcs of the integrated energy system development graph, since not all possible integrated energy system states and transitions can be valid. For this purpose, there are system-wide constraints described above.

The integrated energy system development graph shown in Fig. 3 has four nodes that did not pass the feasibility check (Fig. 4).

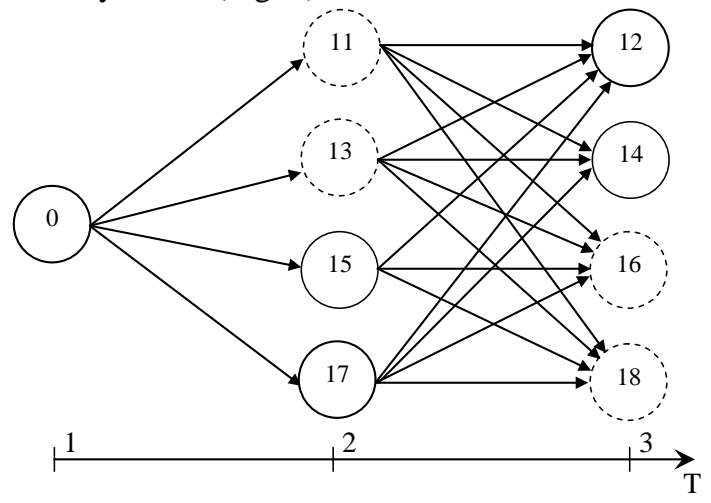

Invalid energy sector state

Fig. 4. Integrated energy system development graph with unfeasible nodes.

The fifth stage is to build a graph containing valid states and transitions. States and transitions that are unreachable from the initial state are determined during the passage from the initial node to the end nodes. Then, the blind states and transitions are determined during the 
reverse passage. It is impossible to build a path from the initial node to the nodes with blind states and transitions at the last time moment. The unfeasible, unreachable and blind states and transitions are removed from the graph which contains possible integrated energy system states and transitions.

In the last stage, a set of system states to form optimal and suboptimal paths can be determined with the algorithm based on the concept of dynamic programming [24].

The graph consisting of valid integrated energy system states and transitions is shown in Fig. 5. It was made from the graph shown in Fig. 4 where an optimal way to ensure minimization of costs of integrated energy system development and operation is presented by the bold lines.

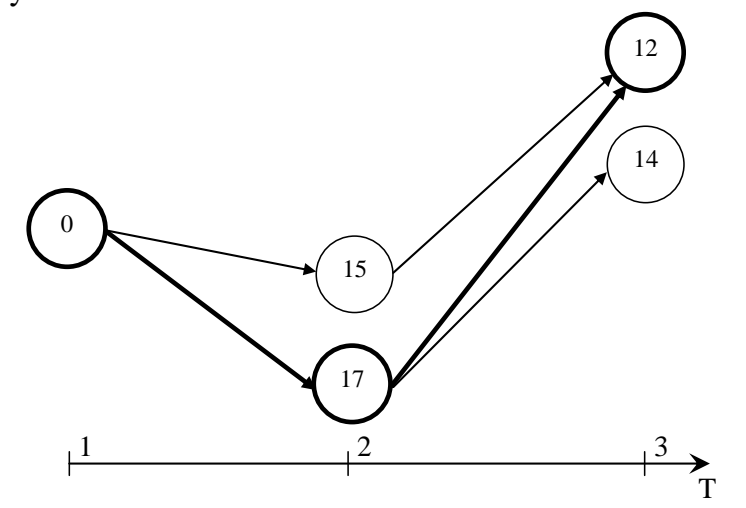

— The optimal way of integrated energy system development

Fig. 5. Integrated energy system optimal graph.

\section{Conclusion}

The advantages of the combinatorial modelling are the clarity and compactness of representation of modelled system development options in the form of a directed graph. The graph clearly illustrates both differences of integrated energy systems development paths and their common states and transitions.

The advantage of this approach is a complete description of the object development options. The traditional approaches to compare the development options based on the multi-criteria methods usually enable researcher to make just a few options. The choice depends on the researcher's intuition and experience. Such a selection, even if it is right, always reflects certain subjectivity and thus depreciates the level of result proof.

The resulting set of the admissible system development paths can be applied in many forecasting tasks where, for example, the uncertainty should be taken into account. Among the feasible system paths, one can choose not merely the best way but also the paths close to it according to research criteria. The carried out researches have shown the possibility of using combinatorial modelling to study the long-term development of integrated energy systems.

\section{Acknowledgements}

The work was carried out within framework of scientific projects III.17.4.1 (No. AAAA-A17-117030310432-9) and III.17.5.1 (No. AAAA-A17-117030310451-0) of the program of fundamental research of the Siberian Branch of the Russian Academy of Sciences. 


\section{References}

1. N. Voropai, V. Stennikov, S. Senderov, E. Barakhtenko, O. Voitov, L. Kovernikova, T. Oshchepkova and L. Semenova, Integrated Infrastructural Energy Systems Challenges for Russia, in Proc. International Conference on Problems of Critical Infrastructures and 6th International Conference on Liberalization and Modernization of Power Systems, eds. Z. A. Styczynski and N. I. Voropai (Saint Petersburg, 2015), pp. 115-121.

2. N. Voropai, V. Stennikov, S. Senderov, E. Barakhtenko, O. Voitov and A. Ustinov, Modeling of Integrated Energy Supply Systems: Main Principles, Model, and Applications, Journal of Energy Engineering 143(5) (2017).

3. C. A. Saldarriaga, R. A. Hincapié and H. Salazar, A holistic approach for planning natural gas and electricity distribution networks, IEEE Transactions on Power Systems 28 (2013) 4052-4063.

4. P. Mancarella, MES (multi-energy systems): An overview of concepts and evaluation models, Energy $\mathbf{6 5}$ (2014) 1-17.

5. M. Chaudry, N. Jenkins, M. Qadrdan and J. Wu, Combined gas and electricity network expansion planning, Applied Energy 113 (2014) 1171-1187.

6. M. T. Rees, J. Wu, N. Jenkins and M. Abeysekera, Carbon constrained design of energy infrastructure for new build schemes, Applied Energy 113 (2014) 1220 1234.

7. F. Kienzle and G. Andersson, Valuing Investments in Multi-Energy Conversion, Storage, and Demand-Side Management Systems Under Uncertainty, IEEE Transactions on Sustainable Energy, 2(2) (2011) 194 202

8. E. A. Martinez Cesena, T. Capuder and P. Mancarella, Flexible Distributed Multienergy Generation System Expansion Planning Under Uncertainty, IEEE Transactions on Smart Grid 7(1) (2016) 348-357.

9. M. Hennen, M. Lampe, P. Voll and A. Bardow, SPREAD-Exploring the decision space in energy systems synthesis, Computers \& Chemical Engineering 106 (2017) 297-308.

10. L. Braunreiter and Y. B. Blumer, Of sailors and divers: How researchers use energy scenarios, Energy Research \& Social Science 40 (2018) 118-126.

11. C. Guivarch, R. Lempert and E. Trutnevyte, Scenario techniques for energy and environmental research: An overview of recent developments to broaden the capacity to deal with complexity and uncertainty, Environmental Modelling \& Software 97 (2017) 201-210.
12. M. G. Morgan and D. W. Keith, Improving the way we think about projecting future energy use and emissions of carbon dioxide, Clim. Change, 90(3) (2008) 189-215

13. P. Berntsen and E. Trutnevyte, Ensuring diversity of national energy scenarios: bottom-up energy system model with Modeling to Generate Alternatives, Energy 126 (2017) 886-898

14. J. H. Guillaume, Arshad, M., A. J. Jakeman, M. Jalava and M. Kummu, Robust discrimination between uncertain management alternatives by iterative reflection on crossover point scenarios: Principles, design and implementations, Environmental Modelling \& Software 83 (2016) 326-343.

15. H. Carlsen, R. Lempert, P. Wikman-Svahn and V. Schweizer, Choosing small sets of policy-relevant scenarios by combining vulnerability and diversity approaches, Environmental Modelling \& Software $\mathbf{8 4}$ (2016) 155-164.

16. L. S. Belyaev, Pay-off matrix technique, Energy 15(7/8) (1990) 631-643.

17. J. Price and I. Keppo, Modelling to generate alternatives: A technique to explore uncertainty in energyenvironment-economy models, Applied energy 195 (2017) 356-369.

18. A. Edelev and I. Sidorov, Combinatorial Modeling Approach to Find Rational Ways of Energy Development with Regard to Energy Security Requirements, in International Conference on Numerical Analysis and Its Applications (Springer, Cham, 2016), pp. 317-324.

19. F. Wiese, S. Hilpert, C. Kaldemeyer and G. Pleßmann, A qualitative evaluation approach for energy system modelling frameworks, Energy, Sustainability and Society 8(1) (2018).

20. S. M. Senderov and A. V. Edelev, Technology to Ensure Energy Security in Development of Russia's Energy Strategy, Energy Systems Research 1(1) (2018) 35-43.

21. A. V. Edelev, V. I. Zorkaltsev, D. Van Binh and N. H. Nam, The Combinatorial Modelling of Vietnam Energy Development, Energy Systems Research 1(1) (2018) 6773.

22. E. Trutnevyte, Does cost optimization approximate the real-world energy transition?, Energy 106 (2016) 182 193.

23. P. Voll, M. Jennings, M. Hennen, N. Shah and A. Bardow, The optimum is not enough: a near-optimal solution paradigm for energy systems synthesis, Energy 82 (2015) 446-456.

24. R. Bellman, Dynamic Programming (Princeton University Press, Princeton, New Jersey, 1957). 